\title{
Modeling the Acoustic Field Generated by a Pulsed Beam for Experimental Proton Range Verification
}

\author{
Michele Riva ${ }^{1}$, Elia A. Vallicelli ${ }^{1, \star}$, Andrea Baschirotto ${ }^{1}$, and Marcello De Matteis ${ }^{1, \star \star}$ \\ ${ }^{1}$ INFN Section and University of Milano Bicocca, piazza della Scienza 3, 20126, Milano, Italy
}

\begin{abstract}
Proton range verification by ionoacoustic wave sensing is a technique under development for applications in hadron therapy as an alternative to nuclear imaging. It provides an acoustic imaging of the proton energy deposition vs. depth using the acoustic wave Time of Flight (ToF). State-of-the-art (based on simulations and experimental results) points out that this detection technique achieves better spatial resolution $(<1 \mathrm{~mm})$ of the proton range comparing with Positron-Emission-Tomography (PET) and prompt gamma ray techniques. This work presents a complete Geant $4 / \mathrm{k}$-Wave model that allows to understand several physical phenomena and to evaluate the key parameters that affect the acoustic field generated by the incident proton radiation.
\end{abstract}

\section{Introduction}

Oncological hadron therapy utilizes proton beams with energies ranging from $65 \mathrm{MeV}$ to $250 \mathrm{MeV}$ for tumor treatment. Protons release very little energy at the interface and most of their energy at the end of their range, in an area called the Bragg Peak (BP). Unlike traditional radiotherapy, this technique allows to deliver most of the dose at the tumor while minimizing damage to the surrounding healthy tissue. Thus, it is very important to accurately determine the Bragg Peak depth to achieve maximum treatment efficacy. Most experimental techniques for proton range verification utilize nuclear imaging techniques such as Positron Emission Tomography (PET), but their precision is limited to few millimeters and cannot be implemented in real-time during the treatment. Ionoacoustic range verification is a promising technique that has been proposed by Y. Hayakawa in 1988 [1]. It exploits the acoustic wave generated by the fast energy deposition in tissues to locate the energy deposition site with sub-millimeter accuracy [2]. However, state-of-the-art experiments lack proper estimation of the impact of sensing electronics on ionoacoustic measurements. in this scenario, a cross-domain model has been developed that describes an incident beam of protons in a water tank, the consequently produced ionoacoustic wave and the electronics used to acquire the signal. The model is composed by Geant4 (proton beam energy deposition), k-Wave (ionoacoustic wave propagation) and Matlab (ultrasound transducer and Analog Front-End model). This model allows to obtain the amplitude of the pressure signal at the end of the acquisition chain and its Signal-to-Noise Ratio (SNR) at different

\footnotetext{
${ }^{1}$ Acknowledgments: This activity has been supported by the Proton Sound Detector (ProSD) project funded by the INFN (Italian National Institute for Nuclear Physics)

^e-mail: e.vallicelli@campus.unimib.it

$\star \star$ e-mail: marcello.dematteis@unimib.it
} 
distances between transducer and Bragg Peak. A case of study with a $19.5 \mathrm{MeV}$ proton beam is here presented, mimicking the setup described in [2], but these techniques can be used also for higher and more clinically relevant energies.

\section{Methods}

\subsection{Geant4 Model}

The Geant4 model represents a mono-energetic and pencil-like $19.5 \mathrm{MeV}$ proton beam using the Electromagnetic Quark Gluon String model with Binary Cascade for primary protons (QGSP BIC EMZ) as Physics List. The pulse length is $50 \mathrm{~ns}$ with $10 \mathrm{~ns}$ gaussian rising and falling edges. The detector consists of a G4Box divided into $6 \mu \mathrm{m}$ sheets transversal to the beam axis using G4PVDivision. The absorber material is G4Water with $997.07 \mathrm{Kg} / \mathrm{m} 3$ density and $78 \mathrm{eV}$ ionization potential. This results in a $4062 \mu \mathrm{m}$ BP depth and around $300 \mu \mathrm{m}$ BP FWHM.

\section{2 k-Wave Model}

The k-Wave model represents the ionoacoustic wave propagation in the water tank. The shape of the source is a $2 \mathrm{~mm}$ diameter (beam radius) and $300 \mu \mathrm{m}$ thick (Bragg Peak FWHM) disk, located $4 \mathrm{~mm}$ from the side surface of the water phantom. The beam energy deposition lasts around 50ns, thus the whole process occurs under stress and thermal confinement conditions, which are respectively $210 \mu \mathrm{s}$ and $0.7 \mathrm{~s}$. This means that the energy deposition is adiabatic and isochoric, and leads to a pressure increase of $270 \mathrm{~Pa}$ for a dose of $2 \mathrm{~Gy}$ at the BP. A series of sensing points lie on the disk axis and acquire the pressure signal at distances from the BP ranging from $100 \mu \mathrm{m}$ to $4.5 \mathrm{~cm}$. The pressure wave amplitude vs distance is shown in Fig.1-Left.

\subsection{Analog Front-End Model}

A commercial Lead Zirconate Titanate (PZT) ultrasound transducer (UST) was modelled in Matlab with a $2 \mathrm{MHz}$ central frequency, $3.68 \mu \mathrm{V} / \mathrm{Pa}$ Sensitivity and $1.3 \mathrm{nV} / \sqrt{\mathrm{Hz}}$ Input Referred Noise (IRN). A dedicated analog front-end (AFE) composed by a $0.9 \mathrm{nV} / \sqrt{\mathrm{Hz}}$ Low-Noise Amplifier, a $2^{\text {nd }}$ order low-pass filter with $2 \mathrm{MHz}$ bandwidth and a 10 bit $50 \mathrm{MS} / \mathrm{s}$ Analog-to-Digital converter has been modelled with Matlab to introduce the impact of electronic noise on the measurement. The AFE model allows to calculate the amplitude in volt of the acquired signal and the noise power, integrated over a $2 \mathrm{MHz}$ bandwidth. Fig.1-Right represents the resulting Signal-to-Noise Ratio at different distances between the transducer and the BP. Evaluating the SNR allows to estimate the precision of the range measurement since it represents the impact of random noise fluctuations on the signal, as was shown in [3] for high-energy scenarios.
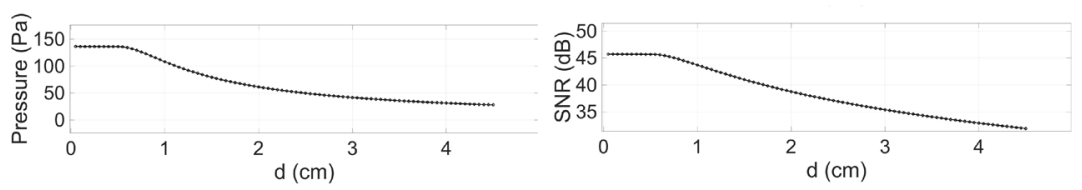

Figure 1. Pressure wave amplitude and Signal-to-Noise Ratio at different distances from the Bragg Peak. 


\section{Results}

The hereby presented cross-domain model has been used to evaluate the impact of the various beam parameters on the intensity of the ionoacoustic signal with the goal to maximize the output SNR for a given dose. Sweep-simulations of beam current rising time and dose per unit time confirmed theoretical expectations that only changes in the deposition rate should generate a pressure wave. Rising time has been varied between $50 \mathrm{~ns}$ and $1000 \mathrm{~ns}$ showing a linear decrease of pressure wave amplitude at $0.5 \mathrm{~cm}$ from 17.7 Pa down to 4.9 Pa (Fig.2-Left). Dose in unit time, which is strictly linked to the beam current intensity, affects the signal in the opposite way: the amplitude increases linearly from $90 \mathrm{mPa}$ up to $948 \mathrm{mPa}$ in the 0.89-82.4 MGy/s range (Fig.2-Right). Moreover, this cross-domain model allowed to obtain optimum design parameters for the AFE stages that minimize the impact of electronics on the SNR and therefore maximize the precision in range estimation for a given pressure signal.

\section{Conclusions}

The evidence that the variation of two beam parameters can improve the amplitude of the signal for a given dose is very important for clinical scenarios where a clear ionoacoustic signal must be obtained within the planned dose budget for the treatment. Higher SNR allows to achieve better spatial resolution and to achieve the desired precision with a reduced dose, thus opening the possibility of real-time in-treatment beam monitoring.
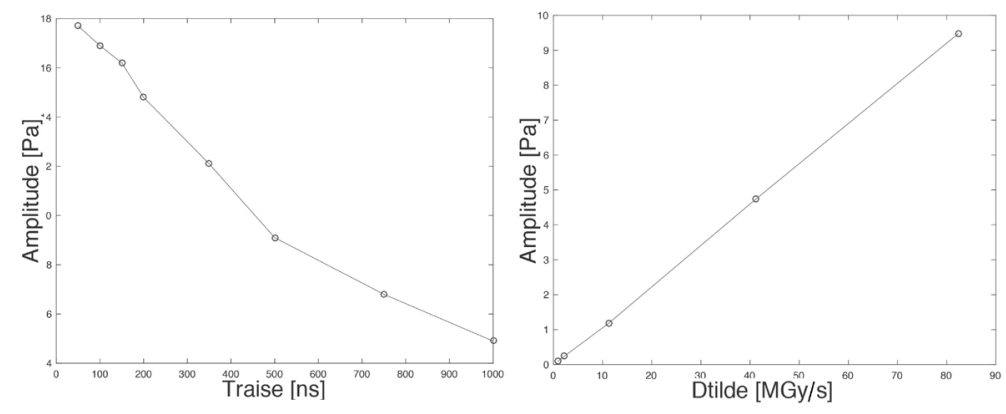

Figure 2. Acoustic signal amplitude vs Rising Time (Left) and vs Deposited Dose per unit time (Right).

\section{References}

[1] Hayakawa, Yoshinori, et al. "Acoustic pulse generated in a patient during treatment by pulsed proton radiation beam." Radiation Oncology Investigations 3.1 (1995): 42-45

[2] Assmann, W., et al. "Ionoacoustic characterization of the proton Bragg peak with submillimeter accuracy." Med physics 42.2 (2015): 567-574

[3] M. Riva, et al. "Acoustic Analog Front-End for Bragg-Peak Detection in Hadron Therapy". In Biomedical Circuits and Systems Conference (BioCAS), 2017 IEEE (pp. 1-4). IEEE 